Aksaray University
Journal of Science and Engineering
e-ISSN: 2587-1277
http://dergipark.gov.tr/asujse
http://asujse.aksaray.edu.tr

Research Article

\title{
Energy and Metric Gauging in the Covariant Theory of Gravitation
}

\author{
Sergey G. Fedosin* \\ Sviazeva str. 22-79, Perm 614088, Perm Krai, Russia
}

-Received Date: 14 Jun 2018 -Accepted Date: 26 Nov 2018

-Published Online: 26 Nov 2018

\begin{abstract}
Relations for the relativistic energy and metric are analyzed inside and outside the body in the framework of the covariant theory of gravitation. The methods of optimal energy gauging and equations for the metric are chosen. It is shown that for the matter inside the body a procedure is required to average the physical quantities, including the cosmological constant and the scalar curvature. For the case of the relativistic uniform system, the cosmological constant and the scalar curvature are explicitly calculated, which turn out to be constant values inside the body and are assumed to be equal to zero outside the body. Comparison of the cosmological constants inside a proton, a neutron star and in the observable Universe allows us to explain the cosmological constant problem arising in the Lambda-CDM model.
\end{abstract}

\section{Keywords}

Cosmological constant, Scalar curvature, Relativistic uniform system, Gravitational field, Acceleration field, Pressure field

\section{INTRODUCTION}

The relativistic energy of the physical system is part of the time component of the fourmomentum of the system and is one of the most important characteristics, along with the momentum. In this case, the energy is determined with an accuracy up to a constant, selected arbitrarily based on the convenience of calculation. Thus, the problem of energy gauging arises in each theory. In the covariant theory of gravitation, the energy is gauged based on the fact that the value of the cosmological constant is proportional with an accuracy to a constant multiplier to the energy density of the matter particles in the proper fields of the system under consideration [1].

*Corresponding Author: Sergey G. Fedosin, fedosin@hotmail.com

2017-2018@Published by AksarayUniversity

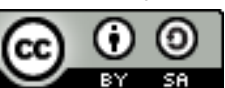


The use of the cosmological constant for energy gauging results in certain changes in the equation for the metric, in which the cosmological constant is present alongside with the scalar curvature. Therefore, we will further analyze both - the expressions for the metric and for the energy.

The purpose of this article is to clarify the question - how the cosmological constant and the scalar curvature in the matter inside bodies should be understood. The point is that, as a rule, representative volumes occupied by typical particles should be selected in the matter, and calculations should be carried out for such particles, including solution of the equation of motion. Applying the method of typical particles implies we need to use the appropriate averaging of the physical quantities acting on such particles. Such quantities as the cosmological constant and the scalar curvature are not exceptions. Thus, they should also be considered as some averaged quantities. As a result of our analysis for the case of the relativistic uniform system, we will calculate the cosmological constant and the scalar curvature inside the body, and will show that they are constant quantities. In addition, we will try to clarify the cosmological constant problem in connection with its inconsistency with the zero energy of the vacuum.

\section{EQUATIONS FOR THE METRIC AND ENERGY}

The use of the principle of least action in the framework of the covariant theory of gravitation leads to the following relation for the metric [1]:

$$
\begin{aligned}
& -2 c k R^{\alpha \beta}+c k R g^{\alpha \beta}-2 c k \Lambda g^{\alpha \beta}= \\
& =D_{\mu} J^{\mu} g^{\alpha \beta}+U^{\alpha \beta}+A_{\mu} j^{\mu} g^{\alpha \beta}+W^{\alpha \beta}+U_{\mu} J^{\mu} g^{\alpha \beta}+B^{\alpha \beta}+\pi_{\mu} J^{\mu} g^{\alpha \beta}+P^{\alpha \beta},
\end{aligned}
$$

where $c$ is the speed of light; $k$ is the constant, which is part of the Lagrangian in the terms with the scalar curvature $R$ and the cosmological constant $\Lambda ; R^{\alpha \beta}$ is the Ricci tensor; $g^{\alpha \beta}$ is the metric tensor; $J^{\mu}$ is the mass four-current; $j^{\mu}$ is the charge four-current; $D_{\mu}, A_{\mu}, U_{\mu}$ and $\pi_{\mu}$ are the four-potentials of the gravitational and electromagnetic fields, the acceleration field and the pressure field, respectively; $U^{\alpha \beta}, W^{\alpha \beta}, B^{\alpha \beta}$ and $P^{\alpha \beta}$ are the stress-energy tensors of these fields, respectively.

Equation (1) can be contracted by means of multiplying by the metric tensor, taking into account that $g_{\alpha \beta} U^{\alpha \beta}=0, \quad g_{\alpha \beta} W^{\alpha \beta}=0, \quad g_{\alpha \beta} B^{\alpha \beta}=0, \quad g_{\alpha \beta} P^{\alpha \beta}=0, \quad g_{\alpha \beta} R^{\alpha \beta}=R$, and in the fourdimensional spacetime $g_{\alpha \beta} g^{\alpha \beta}=4$ : 


$$
c k R-4 c k \Lambda=2 D_{\mu} J^{\mu}+2 A_{\mu} j^{\mu}+2 U_{\mu} J^{\mu}+2 \pi_{\mu} J^{\mu} .
$$

Substitution of (2) into (1) gives an equation for the metric:

$$
R^{\alpha \beta}-\frac{1}{4} R g^{\alpha \beta}=-\frac{1}{2 c k}\left(U^{\alpha \beta}+W^{\alpha \beta}+B^{\alpha \beta}+P^{\alpha \beta}\right) .
$$

Let us take the covariant derivative $\nabla_{\beta}$ of both sides of equation (3):

$$
\nabla_{\beta} R^{\alpha \beta}-\frac{1}{4} g^{\alpha \beta} \nabla_{\beta} R=-\frac{1}{2 c k} \nabla_{\beta}\left(U^{\alpha \beta}+W^{\alpha \beta}+B^{\alpha \beta}+P^{\alpha \beta}\right) .
$$

For the tensors on the right-hand side, the relation $\nabla_{\beta}\left(U^{\alpha \beta}+W^{\alpha \beta}+B^{\alpha \beta}+P^{\alpha \beta}\right)=0$ is valid as an expression of the equation of motion [1]. Consequently, the right-hand side of (4) vanishes. The Ricci tensor and the scalar curvature are part of the Einstein tensor, the covariant derivative of which is equal to zero due to the properties of the curvature tensor and the differential Bianchi identity:

$$
\nabla_{\beta}\left(R^{\alpha \beta}-\frac{1}{2} g^{\alpha \beta} \nabla_{\beta} R\right)=0
$$

From comparison of (5) with the left-hand side of (4), which must also be equal to zero, it follows that $\nabla_{\beta} R=0$. It means that the covariant derivative of the scalar curvature must be equal to zero at any point in space, both inside and outside the system.

In addition to relation (2), which contains the scalar curvature $R$ and the cosmological constant $\Lambda$, there is another relation in [1], which contains these quantities. In particular, for the Hamiltonian and the relativistic energy of the physical system with continuous distribution of matter we found the following:

$$
\begin{aligned}
& E=\frac{1}{c} \int\left(\rho_{0} \psi+\rho_{0 q} \varphi+\rho_{0} \vartheta+\rho_{0} \wp\right) u^{0} \sqrt{-g} d x^{1} d x^{2} d x^{3}- \\
& -\int\left(\begin{array}{l}
c k R-2 c k \Lambda+\frac{c^{2}}{16 \pi G} \Phi_{\mu \nu} \Phi^{\mu \nu}-\frac{1}{4 \mu_{0}} F_{\mu \nu} F^{\mu \nu}- \\
-\frac{c^{2}}{16 \pi \eta} u_{\mu \nu} u^{\mu \nu}-\frac{c^{2}}{16 \pi \sigma} f_{\mu \nu} f^{\mu \nu}
\end{array}\right) \sqrt{-g} d x^{1} d x^{2} d x^{3} .
\end{aligned}
$$

In (6) $\rho_{0}$ and $\rho_{0 q}$ denote the invariant densities of mass and charge, respectively; $\psi, \varphi, \vartheta$ and $\wp$ are the scalar potentials of the gravitational and electromagnetic fields, the acceleration field and the pressure field, respectively; $\Phi_{\mu \nu}, F_{\mu \nu}, u_{\mu \nu}$ and $f_{\mu v}$ are the tensors of these fields, respectively; $u^{0}$ is the time component of the four-velocity of the matter unit; $g$ is the metric tensor determinant; $d x^{1} d x^{2} d x^{3}$ is the product of the differentials of space coordinates; $G$ is the 
gravitational constant; $\mu_{0}$ is the magnetic constant; $\eta$ is the acceleration field constant; $\sigma$ is the pressure field constant.

\section{GAUGING OUTSIDE THE BODY}

We will use (6) to calculate the contribution into the system's energy outside the body, where there is no matter and there are only the gravitational and electromagnetic fields, so it is sufficient to take into account only the second integral. In this case, the mass and charge fourcurrents are equal to zero and the condition $R_{o}=4 \Lambda_{o}$ remains in (2), where the symbol $o$ refers to the quantities outside the body. Under this condition, the contribution into the energy (6) outside the body will be:

$$
E_{o}=\int\left(-2 c k \Lambda_{o}-\frac{c^{2}}{16 \pi G} \Phi_{\mu \nu} \Phi^{\mu \nu}+\frac{1}{4 \mu_{0}} F_{\mu \nu} F^{\mu \nu}\right) \sqrt{-g} d x^{1} d x^{2} d x^{3}
$$

It is convenient to assume that in (7) the cosmological constant $\Lambda_{o}=0$, that is, the contribution into the relativistic energy in the volume outside the system depends neither on the scalar curvature nor on the cosmological constant. Then the condition $R_{o}=4 \Lambda_{o}$ implies the equality $R_{o}=0$. As a result, we obtain the equality $\nabla_{\beta} R_{o}=0$, which follows from (4) and (5).

The fact that both the scalar curvature $R_{o}$ and the cosmological constant $\Lambda_{o}$ are assumed to be zero outside the body was used in [2] to calculate the metric tensor components and to simplify the equation for the metric (3) to the following form:

$$
R^{\alpha \beta}=-\frac{1}{2 c k}\left(U^{\alpha \beta}+W^{\alpha \beta}\right) .
$$

\section{GAUGING INSIDE THE BODY}

We will now pass on to the situation inside the body, where all the stress-energy tensors on the right-hand side of the equation for the metric (3) are non-zero. Since according to (4) and (5) the condition $\nabla_{\beta} R_{i}=0$ must hold, where the symbol $i$ refers to the quantities in the matter inside the body, then after applying the covariant derivative to all the terms in (2) the following remains:

$$
\nabla_{\beta}\left(2 c k \Lambda_{i}+D_{\mu} J^{\mu}+A_{\mu} j^{\mu}+U_{\mu} J^{\mu}+\pi_{\mu} J^{\mu}\right)=0 .
$$

We will now substitute the scalar curvature from (2) into the expression for the energy (6): 


$$
\begin{aligned}
& E_{i}=\frac{1}{c} \int\left(\rho_{0} \psi+\rho_{0 q} \varphi+\rho_{0} \vartheta+\rho_{0} \wp\right) u^{0} \sqrt{-g} d x^{1} d x^{2} d x^{3}- \\
& -\int\left(\begin{array}{l}
2 c k \Lambda_{i}+2 D_{\mu} J^{\mu}+2 A_{\mu} j^{\mu}+2 U_{\mu} J^{\mu}+2 \pi_{\mu} J^{\mu}+ \\
+\frac{c^{2}}{16 \pi G} \Phi_{\mu \nu} \Phi^{\mu \nu}-\frac{1}{4 \mu_{0}} F_{\mu \nu} F^{\mu \nu}-\frac{c^{2}}{16 \pi \eta} u_{\mu \nu} u^{\mu \nu}-\frac{c^{2}}{16 \pi \sigma} f_{\mu \nu} f^{\mu \nu}
\end{array}\right) \sqrt{-g} d x^{1} d x^{2} d x^{3} .
\end{aligned}
$$

We find the cosmological constant $\Lambda_{i}$ in two relations - in (9) and in (10). In (9), the covariant derivative $2 c k \nabla_{\beta} \Lambda_{i}$ must behave with an accuracy up to a sign like the covariant derivative $\nabla_{\beta}\left(D_{\mu} J^{\mu}+A_{\mu} j^{\mu}+U_{\mu} J^{\mu}+\pi_{\mu} J^{\mu}\right)$. And in (10) the term $2 c k \Lambda_{i}$ is some additional energy density. The choice of the value of $\Lambda_{i}$ for gauging purposes is not initially limited by anything, except that it must be invariant with respect to the covariant transformations of coordinates and time. For convenience we will use the simplest variant, which significantly simplifies the expression for the energy.

Just as in [1], we will suppose that the cosmological constant $\Lambda_{i}$ in the matter inside the body is such that the following relation would hold:

$$
-c k \Lambda_{i}=D_{\mu} J^{\mu}+A_{\mu} j^{\mu}+U_{\mu} J^{\mu}+\pi_{\mu} J^{\mu} .
$$

Then, according to (10) and (11), the energy in the space inside the body occupied by the matter and fields will no longer depend on the cosmological constant:

$$
\begin{aligned}
& E_{i}=\frac{1}{c} \int\left(\rho_{0} \psi+\rho_{0 q} \varphi+\rho_{0} \vartheta+\rho_{0} \wp\right) u^{0} \sqrt{-g} d x^{1} d x^{2} d x^{3}- \\
& -\int\left(\frac{c^{2}}{16 \pi G} \Phi_{\mu \nu} \Phi^{\mu \nu}-\frac{1}{4 \mu_{0}} F_{\mu \nu} F^{\mu \nu}-\frac{c^{2}}{16 \pi \eta} u_{\mu \nu} u^{\mu \nu}-\frac{c^{2}}{16 \pi \sigma} f_{\mu \nu} f^{\mu \nu}\right) \sqrt{-g} d x^{1} d x^{2} d x^{3} .
\end{aligned}
$$

Assuming that the cosmological constant outside the body is equal to zero because of the absence of matter there, $\Lambda_{o}=0$, we will compare the relations for the energy (7) and (12). From these relations we can see that the general expression for the energy is (12), in which the energy $E_{i}$ must be replaced with $E$. The system's energy $E$ with the right-hand side in the form of (12) was derived by us earlier in [1].

The denser the cosmic object is, the higher is the energy density of the particles in the field potentials on the right-hand side of (11), and the greater is the cosmological constant $\Lambda_{i}$ inside the body. Since (11) and (2) imply the relation $R_{i}=2 \Lambda_{i}$, then the scalar curvature $R_{i}$ inside the bodies is not equal to zero and varies proportionally to the cosmological constant $\Lambda_{i}$. Consequently, in denser bodies the scalar curvature has greater value. 
We will express the four-potentials of the fields in (11) in terms of the respective scalar and vector potentials of these fields: $D_{\mu}=\left(\frac{\psi}{c},-\mathbf{D}\right)$ for the gravitational field, $A_{\mu}=\left(\frac{\varphi}{c},-\mathbf{A}\right)$ for the electromagnetic field, $U_{\mu}=\left(\frac{\vartheta}{c},-\mathbf{U}\right)$ for the acceleration field, $\pi_{\mu}=\left(\frac{\wp}{c},-\boldsymbol{\Pi}\right)$ for the pressure field. In the limit of the special theory of relativity, the four-currents have the following form: $J^{\mu}=\rho_{0} u^{\mu}=\rho_{0}(\gamma c, \gamma \mathbf{v}), j^{\mu}=\rho_{0 q} u^{\mu}=\rho_{0 q}(\gamma c, \gamma \mathbf{v})$, where $\gamma$ is the Lorentz factor of the particle of the moving and continuously distributed matter of the system, $\mathbf{v}$ is the velocity of the particle's motion. This gives the following:

$$
-c k \Lambda_{i}=\gamma \rho_{0} \psi-\gamma \rho_{0} \mathbf{D} \cdot \mathbf{v}+\gamma \rho_{0 q} \varphi-\gamma \rho_{0 q} \mathbf{A} \cdot \mathbf{v}+\gamma \rho_{0} \vartheta-\gamma \rho_{0} \mathbf{U} \cdot \mathbf{v}+\gamma \rho_{0} \wp-\gamma \rho_{0} \boldsymbol{\Pi} \cdot \mathbf{v} .
$$

Relations (11) and (13) must hold true not only for the matter inside the body, but also for the matter in such a state, when this matter has not yet aggregated into a closely connected system and was in the form of particles distant from each other. In the latter case, the cosmological constant and the scalar curvature inside individual particles have their proper values.

In the limit of low velocities we can neglect the terms containing the velocity $\mathbf{v}$ of the particles and the vector potentials $\mathbf{D}, \mathbf{A}, \mathbf{U}$ and $\boldsymbol{\Pi}$. Then in (13) the Lorentz factor is $\gamma \approx 1$ and only the terms with scalar field potentials are left. For the particles scattered at infinity in cosmic space we can assume that these potentials arise only from the particles' proper fields and are the potentials averaged over the volume of particles. In this case, according to [3], $\vartheta \approx \gamma_{c} c^{2}$, where $\gamma_{c}$ is the Lorentz factor for the matter at the center of the particles. Denoting the cosmological constant for individual particles in cosmic space by $\Lambda_{0}$ we can write:

$$
-c k \Lambda_{0} \approx \rho_{0} \psi+\rho_{0 q} \varphi+\gamma_{c} \rho_{0} c^{2}+\rho_{0} \wp .
$$

From (11) and (14) it follows that in the absence of matter the cosmological constant vanishes. This is consistent with the fact that in Section 3 we assumed that the cosmological constant outside the body is equal to zero.

According to (14), $\Lambda_{0}$ is defined by the rest energy density of the particles with a certain addition from the energy density of the particles in the gravitational and electromagnetic fields and in the pressure field in the matter. Now we can average $\Lambda_{0}$ over the entire space, as well as the mass density $\rho_{0}$ and the charge density $\rho_{0 q}$, without changing the values of the field potentials. To do this, we will take into account that in the first approximation the product $\gamma_{c} \rho_{0}$ is the ratio of the particle's mass to the particle's volume as some average density. Averaging 
over the entire space will take place if we distribute the particle's mass over the entire volume, which, on the average, can be attributed to one particle in cosmic space. In this case $\gamma_{c} \rho_{0}$ is changed to $\bar{\rho}_{0}$ and $\Lambda_{0}$ to $\bar{\Lambda}_{0}$. Leaving in (14) only the rest energy as the basic term due to its value, we can approximately write:

$$
-c k \bar{\Lambda}_{0} \approx \bar{\rho}_{0} c^{2}
$$

Using the definition in the following form: $k=-\frac{c^{3}}{16 \pi G \beta}$, where $\beta$ is a constant of the order of unity, we find the averaged value $\bar{\Lambda}_{0} \approx \frac{16 \pi G \bar{\rho}_{0}}{c^{2}}$. Substituting instead of $\bar{\Lambda}_{0}$ the estimate of the cosmological constant $\Lambda \approx 10^{-52} \mathrm{~m}^{-2}$ according to the Lambda-CDM model [4], we find the corresponding density: $\bar{\rho}_{0} \approx 2.7 \times 10^{-27} \mathrm{~kg} / \mathrm{m}^{3}$, which is close enough to the observed average mass density of the matter.

If we consider the proton, which is stable in all respects, as the basic particle in cosmic space, then we can estimate the cosmological constant $\Lambda_{0}$ for it in (14). To do this, instead of $\gamma_{c} \rho_{0}$ we should use the average proton density of the order of $\rho_{p}=6 \times 10^{17} \mathrm{~kg} / \mathrm{m}^{3}$ with its radius $8.73 \times 10^{-16} \mathrm{~m}$, according to [5]. This gives the value $\Lambda_{0} \approx \frac{16 \pi G \rho_{p}}{c^{2}} \approx 2.2 \times 10^{-8} \mathrm{~m}^{-2}$, which is 44 orders of magnitude greater than the cosmological constant $\Lambda \approx 10^{-52} \mathrm{~m}^{-2}$ averaged over the entire cosmic space.

The next step can be made by taking into account the strong gravitation described in [6,7], and assumed as the basis for describing the strong interaction at the hadron level. If we substitute the gravitational constant $G$ with the strong gravitational constant $G_{a}=1.514 \times 10^{29} \mathrm{~m}^{3} /\left(\mathrm{kg} \cdot \mathrm{s}^{2}\right)$ according to [8], then the corresponding cosmological constant for the proton will equal $\Lambda_{p} \approx \frac{16 \pi G_{a} \rho_{p}}{c^{2}} \approx 5.1 \times 10^{31} \mathrm{~m}^{-2}$. The relation for the scalar curvature $R_{i}=2 \Lambda_{i}$ for the matter inside the proton will be written as $R_{p}=2 \Lambda_{p} \approx 10^{32} \mathrm{~m}^{-2}$. Assuming in the first approximation that the spacetime inside the proton has constant curvature, we will estimate the radius of curvature, based on the expression in [9], which relates the scalar curvature and the radius of curvature: $r_{p} \approx \sqrt{\frac{12}{R_{p}}}=3.4 \times 10^{-16} \mathrm{~m}$. The value $r_{p}$, calculated in the field of strong gravitation, is of the order of the proton radius. 


\section{AVERAGING OF PHYSICAL QUANTITIES INSIDE THE BODY}

While estimating the matter parameters the usual procedure is to single out particles or volume elements of such sizes, that they could characterize on the average the basic properties of the matter. For example, in a crystalline solid body a typical element is a crystal cell, so that the whole body can be divided into a number of such cells. If we consider the intervals between the typical particles of matter to be small, then to such matter in the form of liquid we can apply the approximation of continuous medium. In this case, the particles remain independent to some extent and can move at different velocities. However, due to the close interaction of particles, in each stationary system certain dependences of physical quantities on the coordinates and time are established, which characterize the system on the average. We will assume that the typical particles of the system have exactly such parameters, which define the average physical quantities in the matter. Actually this means that in all equations used to describe the matter all quantities refer to typical particles.

We will next consider a non-rotating body of a spherical shape, which represents a physical system of closely interacting particles and fields, held in equilibrium by gravitation, and will use a relativistic uniform model to describe all physical quantities.

Within the framework of the special theory of relativity, the covariant derivatives are replaced with the four-gradient, and this relation follows from (11) and (9):

$$
-c k \partial_{\beta} \Lambda_{i}=\partial_{\beta}\left(D_{\mu} J^{\mu}+A_{\mu} j^{\mu}+U_{\mu} J^{\mu}+\pi_{\mu} J^{\mu}\right)=0 .
$$

On the right-hand side of (15) we will express the products of the four-potentials of the fields by the four-currents in the same way as it was done in (13). For the physical system under consideration, the vector field potentials $\mathbf{D}, \mathbf{A}, \mathbf{U}$ and $\boldsymbol{\Pi}$ averaged over a sufficient number of typical particles vanish due to the chaotic motion of these particles, and the Lorentz factor of the particles is the quantity $\gamma^{\prime}$ as a function of the current radius. Consequently, in (13), in a first approximation we can neglect the terms with the vector potentials, and the following remains in (15):

$$
-c k \partial_{\beta} \Lambda_{i}=\partial_{\beta}\left[\left(\rho_{0} \psi+\rho_{0 q} \varphi+\rho_{0} \vartheta+\rho_{0} \wp\right) \gamma^{\prime}\right]=0 .
$$

The scalar potentials of the fields inside the sphere with the radius $a$ were found in [3], [10]:

$$
\begin{gathered}
\bar{\psi}=\frac{G c^{2} \gamma_{c}}{\eta} \cos \left(\frac{a}{c} \sqrt{4 \pi \eta \rho_{0}}\right)-\frac{G c^{3} \gamma_{c}}{r \eta \sqrt{4 \pi \eta \rho_{0}}} \sin \left(\frac{r}{c} \sqrt{4 \pi \eta \rho_{0}}\right) \approx \frac{2 \pi G \gamma_{c} \rho_{0}\left(r^{2}-3 a^{2}\right)}{3}, \\
\bar{\varphi}=-\frac{\rho_{0 q} c^{2} \gamma_{c}}{4 \pi \varepsilon_{0} \eta \rho_{0}} \cos \left(\frac{a}{c} \sqrt{4 \pi \eta \rho_{0}}\right)+\frac{\rho_{0 q} c^{3} \gamma_{c}}{4 \pi \varepsilon_{0} r \eta \rho_{0} \sqrt{4 \pi \eta \rho_{0}}} \sin \left(\frac{r}{c} \sqrt{4 \pi \eta \rho_{0}}\right) \approx-\frac{\gamma_{c} \rho_{0 q}\left(r^{2}-3 a^{2}\right)}{6 \varepsilon_{0}},
\end{gathered}
$$




$$
\begin{gathered}
\bar{\vartheta}=\gamma^{\prime} c^{2}=\frac{c^{3} \gamma_{c}}{r \sqrt{4 \pi \eta \rho_{0}}} \sin \left(\frac{r}{c} \sqrt{4 \pi \eta \rho_{0}}\right) \approx c^{2} \gamma_{c}-\frac{2 \pi \eta \rho_{0} r^{2} \gamma_{c}}{3}, \\
\bar{\wp}=\wp_{c}-\frac{\sigma c^{2} \gamma_{c}}{\eta}+\frac{\sigma c^{3} \gamma_{c}}{r \eta \sqrt{4 \pi \eta \rho_{0}}} \sin \left(\frac{r}{c} \sqrt{4 \pi \eta \rho_{0}}\right) \approx \wp_{c}-\frac{2 \pi \sigma \rho_{0} r^{2} \gamma_{c}}{3},
\end{gathered}
$$

where $r$ is the current radius, $\varepsilon_{0}$ is the electric constant, $\wp_{c}$ is the pressure field potential at the center of the sphere, and $\gamma_{c}$ is the Lorentz factor at the center of the sphere.

The scalar potentials (17) depend on the current radius and give the averaged values as a consequence of interaction of the entire set of typical particles. Before substituting these potentials into (16), equation (16) should be averaged over the volume of a typical particle. This also means that when using the cosmological constant and the scalar curvature inside the body, these quantities should be considered as some averaged quantities.

If we denote by $V_{0 p}$ the proper volume of a typical particle and by $V_{p}$ the apparent volume of a moving particle from the viewpoint of an observer, who is stationary relative to the body, then averaging of the left-hand side of (16) over the volume of the moving particle yields:

$$
-c k \partial_{\beta}\left(\frac{1}{V_{0 p}} \int \Lambda_{i} d V_{p}\right)=-c k \partial_{\beta} \bar{\Lambda}
$$

where $\bar{\Lambda}$ is the averaged scalar curvature inside the body at the location of the given typical particle.

For the right-hand side of (16), averaging leads to the following:

$$
\frac{1}{V_{0 p}} \partial_{\beta}\left(\int\left[\left(\rho_{0} \psi+\rho_{0 q} \varphi+\rho_{0} \vartheta+\rho_{0} \wp\right) \gamma^{\prime}\right] d V_{p}\right)=0 .
$$

The volume element $d V_{p}$ in the integrals (18) and (19) is the volume element of a moving particle from the viewpoint of an observer, who is stationary relative to the body, so that $V_{p}=\int d V_{p}$. The value $\frac{1}{V_{0 p}}$ in the approximation of the special theory of relativity is a constant value for the particle under consideration, and therefore in (19) it was taken outside the derivative sign.

Since the whole set of particles densely fill the sphere, for the given observer the sum of the volumes of all moving particles should give the volume of the sphere: $V_{s}=\sum_{p} V_{p}$. Hence it follows that the volume element $d V_{p}$ can also be considered as the volume element $d V$ of a 
fixed sphere, so that by summing all these volume elements this observer can determine the volume of the sphere.

On the other hand, the typical particle chosen by us moves at a certain averaged velocity $\mathbf{v}$ and with the corresponding Lorentz factor $\gamma^{\prime}$. As a result, if a particle at rest has the volume $V_{0 p}$, then a moving particle, from the point of view of the theory of relativity, has the reduced volume $V_{p}$, while $V_{0 p}=\gamma^{\prime} V_{p}$, as well as $d V_{0 p}=\gamma^{\prime} d V_{p}=\gamma^{\prime} d V$, taking into account the equation $d V_{p}=d V$. This fact was used in [11] when considering the virial theorem.

In (19), the quantities $\rho_{0} \gamma^{\prime} d V_{p}=\rho_{0} d V_{0 p}=d m_{p}$ and $\rho_{0 q} \gamma^{\prime} d V_{p}=\rho_{0 q} d V_{0 p}=d q_{p}$ represent the elements of mass and charge of the particle. With this in mind, equation (19) can be rewritten using the averaged scalar potentials (17) of the fields inside the sphere:

$$
\frac{1}{V_{0 p}} \partial_{\beta}\left[m_{p}(\bar{\psi}+\bar{\vartheta}+\bar{\wp})+q_{p} \bar{\varphi}\right]=\partial_{\beta}\left[\rho_{0}(\bar{\psi}+\bar{\vartheta}+\bar{\wp})+\rho_{0 q} \bar{\varphi}\right]=0 \text {. }
$$

Expression (20) is a certain four-vector, each component of which must be zero. The time component of this four-vector vanishes, since the potentials in the stationary sphere do not depend on time, just as the Lorentz factor $\gamma^{\prime}$ of the particles. It remains to consider the space components in (20), for which we will use the relation for the field coefficients, derived in [12] from the equation of motion of matter:

$$
\eta+\sigma=G-\frac{\rho_{0 q}^{2}}{4 \pi \varepsilon_{0} \rho_{0}^{2}}=G-\frac{q_{p}^{2}}{4 \pi \varepsilon_{0} m_{p}^{2}} .
$$

If we substitute the potentials (17) into (20) and take into account (18) and (21), then we see that indeed for the time and space components of the four-gradient of the averaged cosmological constant the following relation holds true:

$$
-c k \partial_{\beta} \bar{\Lambda}=\partial_{\beta}\left[\rho_{0}(\bar{\psi}+\bar{\vartheta}+\bar{\wp})+\rho_{0 q} \bar{\varphi}\right]=0 .
$$

\section{COSMOLOGICAL CONSTANT AND SCALAR CURVATURE INSIDE THE BODY}

Since (11) and (2) imply the relation $R_{i}=2 \Lambda_{i}$, a similar equation must also exist for the averaged quantities. This means that the scalar curvature inside the body must also be averaged and transformed into $\bar{R}$, while the relations $\bar{R}=2 \bar{\Lambda}$ and $\nabla_{\beta} \bar{R}=0$ must be satisfied. Accordingly, equation for the metric (3) inside the body must be written for the averaged quantities: 


$$
\bar{R}^{\alpha \beta}-\frac{1}{4} \bar{R} \bar{g}^{\alpha \beta}=-\frac{1}{2 c k}\left(\bar{U}^{\alpha \beta}+\bar{W}^{\alpha \beta}+\bar{B}^{\alpha \beta}+\bar{P}^{\alpha \beta}\right)
$$

We can assume that relation (22) was obtained by averaging (11) and subsequent taking of the four-gradient. Removing the sign of the four-gradient $\partial_{\beta}$ from (22), taking into account (17) and (21), we find the following inside the body:

$$
\begin{aligned}
& -c k \bar{\Lambda}=\rho_{0}(\bar{\psi}+\bar{\vartheta}+\bar{\gamma})+\rho_{0 q} \bar{\varphi}= \\
& =\frac{G \rho_{0} c^{2} \gamma_{c}}{\eta} \cos \left(\frac{a}{c} \sqrt{4 \pi \eta \rho_{0}}\right)-\frac{\rho_{0 q}^{2} c^{2} \gamma_{c}}{4 \pi \varepsilon_{0} \eta \rho_{0}} \cos \left(\frac{a}{c} \sqrt{4 \pi \eta \rho_{0}}\right)+\rho_{0} \wp_{c}-\frac{\sigma \rho_{0} c^{2} \gamma_{c}}{\eta} .
\end{aligned}
$$

Let us now use the value of the system's total charge $q_{b}$, as well as the value of the system's gravitational mass $m_{g}$, which, according to [10], is equal to the total mass of the system's particles $m_{b}$ :

$$
\begin{gathered}
q_{b}=\int d q=\int \rho_{0 q} \gamma^{\prime} d V=\frac{\rho_{0 q} c^{2} \gamma_{c}}{\eta \rho_{0}}\left[\frac{c}{\sqrt{4 \pi \eta \rho_{0}}} \sin \left(\frac{a}{c} \sqrt{4 \pi \eta \rho_{0}}\right)-a \cos \left(\frac{a}{c} \sqrt{4 \pi \eta \rho_{0}}\right)\right] . \\
m_{g}=m_{b}=\int d m=\int \rho_{0} \gamma^{\prime} d V=\frac{c^{2} \gamma_{c}}{\eta}\left[\frac{c}{\sqrt{4 \pi \eta \rho_{0}}} \sin \left(\frac{a}{c} \sqrt{4 \pi \eta \rho_{0}}\right)-a \cos \left(\frac{a}{c} \sqrt{4 \pi \eta \rho_{0}}\right)\right] .
\end{gathered}
$$

Expressing in (24) the corresponding cosines in terms of the mass $m_{g}$ and the charge $q_{b}$, and then expanding the sine according to the rule $\sin x \approx x-\frac{x^{3}}{6}$, in view of (21) we find:

$$
\begin{aligned}
& -c k \bar{\Lambda}=\frac{G \rho_{0}}{a}\left[\frac{c^{3} \gamma_{c}}{\eta \sqrt{4 \pi \eta \rho_{0}}} \sin \left(\frac{a}{c} \sqrt{4 \pi \eta \rho_{0}}\right)-m_{g}\right]- \\
& -\frac{\rho_{0 q}}{4 \pi \varepsilon_{0} a}\left[\frac{\rho_{0 q} c^{3} \gamma_{c}}{\eta \rho_{0} \sqrt{4 \pi \eta \rho_{0}}} \sin \left(\frac{a}{c} \sqrt{4 \pi \eta \rho_{0}}\right)-q_{b}\right]+\rho_{0} \wp_{c}-\frac{\sigma \rho_{0} c^{2} \gamma_{c}}{\eta} \approx \\
& \approx-\frac{G m_{g} \rho_{0}}{a}-\frac{G m \rho_{0} \gamma_{c}}{2 a}+\rho_{0} c^{2} \gamma_{c}+\frac{q_{b} \rho_{0 q}}{4 \pi \varepsilon_{0} a}+\frac{q \rho_{0 q} \gamma_{c}}{8 \pi \varepsilon_{0} a}+\rho_{0} \wp_{c} .
\end{aligned}
$$

In (25) the auxiliary mass $m=\frac{4 \pi \rho_{0} a^{3}}{3}$ and the auxiliary charge $q=\frac{4 \pi \rho_{0 q} a^{3}}{3}$ are used.

Introducing then the scalar potential of the gravitational field $\psi_{a}=-\frac{G m_{g}}{a}$ and the scalar potential of the electric field $\varphi_{a}=\frac{q_{b}}{4 \pi \varepsilon_{0} a}$ on the surface of the body at $r=a$, we obtain: 


$$
-c k \bar{\Lambda} \approx \rho_{0} \psi_{a}-\frac{G m \rho_{0} \gamma_{c}}{2 a}+\rho_{0} c^{2} \gamma_{c}+\rho_{0 q} \varphi_{a}+\frac{q \rho_{0 q} \gamma_{c}}{8 \pi \varepsilon_{0} a}+\rho_{0} \wp_{c} .
$$

Thus, the averaged cosmological constant $\bar{\Lambda}$ is non-zero and is a constant value inside the fixed body. The same is true for the averaged scalar curvature $\bar{R}$ in (23) since $\bar{R}=2 \bar{\Lambda}$. In this case the required condition $\nabla_{\beta} \bar{R}=0$ is met automatically.

Actually relations (25-26) for the body repeat relation (14) for an individual particle. However, (25-26) are much more informative. In particular, from (26) it follows that the cosmological constant $\bar{\Lambda}$ depends on the scalar potentials of the fields. In this case, the potential $\wp_{c}$ of the pressure field and the potential $\vartheta_{c}=c^{2} \gamma_{c}$ of the acceleration field are taken at the center of the body, but the potentials of the gravitational field $\psi_{a}$ and the electric field $\varphi_{a}$ are taken not at the center, but on the surface of the body. The latter is associated with a special way of gauging the energy and potentials of the gravitational and electric fields - they are gauged so that as the distance to infinity increases, they vanish.

We can also specify the values of the quantities $\gamma_{c}$ and $\wp_{c}$ so that in (26) all the quantities were determined more precisely. In [11], the expression was found for the square of the particles' velocities $v_{c}^{2}$ at the center of a spherical body, with the help of which we can estimate the value of the corresponding Lorentz factor:

$$
\gamma_{c}=\frac{1}{\sqrt{1-v_{c}^{2} / c^{2}}} \approx 1+\frac{v_{c}^{2}}{2 c^{2}}+\frac{3 v_{c}^{4}}{8 c^{4}} \approx 1+\frac{3 \eta m}{10 a c^{2}}\left(1+\frac{9}{2 \sqrt{14}}\right)+\frac{27 \eta^{2} m^{2}}{200 a^{2} c^{4}}\left(1+\frac{9}{2 \sqrt{14}}\right)^{2} .
$$

In this case, the scalar potential of the pressure field at the center of the body is approximately equal to:

$$
\wp_{c} \approx \frac{3 \sigma m}{10 a}\left(1+\frac{9}{2 \sqrt{14}}\right),
$$

and the constant of the acceleration field $\eta$ and the constant of the pressure field $\sigma$ are expressed by the formulas:

$$
\eta=\frac{3}{5}\left(G-\frac{\rho_{0 q}^{2}}{4 \pi \varepsilon_{0} \rho_{0}^{2}}\right), \quad \sigma=\frac{2}{5}\left(G-\frac{\rho_{0 q}^{2}}{4 \pi \varepsilon_{0} \rho_{0}^{2}}\right) .
$$

\section{CONCLUSION}

According to the conclusions in Section 3, outside the body both the cosmological constant $\Lambda_{o}$ and the scalar curvature $R_{o}$ are assumed to be zero. This leads to expression (7) for the 
contribution of the field energy outside the body into the total relativistic energy of the system and to equation for the external metric (8).

As for the situation inside the body, it is necessary to perform an operation of averaging the physical quantities in such a way that they would correspond to the typical particles, which most fully characterize the physical system. The order of averaging of the physical quantities is described in Section 5. After averaging, the scalar curvature and the cosmological constant inside the body are connected by the relation $\bar{R}=2 \bar{\Lambda}$, where they are constant quantities. In (25-26) the cosmological constant $\bar{\Lambda}$ is expressed in terms of the potentials of all the fields existing in the system. The same is true for the scalar curvature $\bar{R}$, in this case the potentials of the acceleration field and the pressure field are taken at the center of the body, and the potentials of the gravitational and electric fields are taken on the surface of the body.

For gravitationally bound bodies the second most important value after the rest energy is the gravitational energy. It follows from (26) that if the coefficient $k$ is negative, then as the mass density $\rho_{0}$ inside the sphere with the constant radius $a$ increases, then due to the increase in the rest energy density both the cosmological constant $\bar{\Lambda}$ and the scalar curvature $\bar{R}$ increase as well. But since the absolute value of the gravitational energy density inside the body increases proportionally to the square of the mass density, this somewhat slows down the increase of the $\bar{\Lambda}$ and $\bar{R}$.

Taking into account $k=-\frac{c^{3}}{16 \pi G \beta}$ we will apply (26) to estimate the cosmological constant in the matter inside a neutron star, leaving on the right-hand side only the rest energy density: $\bar{\Lambda}_{s} \approx \frac{16 \pi G \rho_{s}}{c^{2}} \approx 1.4 \times 10^{-8} \mathrm{~m}^{-2}$. Here we used the average mass density of the star of the order of $\rho_{s}=3.7 \times 10^{17} \mathrm{~kg} / \mathrm{m}^{3}$ with its radius of $12 \mathrm{~km}$ and the mass of a typical star of 1.35 solar masses. Passing on to the scalar curvature with the help of the equation $\bar{R}_{s}=2 \bar{\Lambda}_{s}$, we can estimate the radius of static spacetime curvature inside the star as in a spherical Riemannian space: $r_{s} \approx \sqrt{\frac{12}{\bar{R}_{s}}}=2.1 \times 10^{4} \mathrm{~m}$

As a rule, particles are located inside the bodies in such a way that some gaps remain between the particles. This leads to the fact that in massive objects the average densities of mass and energy do not exceed the corresponding values of the densities inside the particles. As a 
consequence, in the process of transition to such objects the values of the averaged cosmological constant and the scalar curvature decrease. In addition, the cosmological constant in each system turns out to be limited to a certain value, which is, according to (11), proportional to the rest energy density of this matter with regard to the proper fields, and which is a certain reference point in gauging of the relativistic energy (12).

We can compare our calculation for the neutron star and calculation for the proton made in Section 4. According to the theory of infinite nesting of matter [13], these objects are analogous to each other in many respects, while for the proton we used both the ordinary gravitational constant and the strong gravitational constant. Both for the neutron star, and using the strong gravitational constant for the proton, we obtained that the radius of the spacetime curvature does not differ much in the order of magnitude from the radius of the corresponding object. The ratio of the specified radii is the following:

$$
\frac{r_{s}}{r_{p}}=\sqrt{\frac{R_{p}}{\bar{R}_{s}}}=6 \times 10^{19}
$$

We will now use the coefficients of similarity between the neutron star and the proton. The ratio of the star's mass of to the proton's mass gives the coefficient of similarity in mass $\Phi=1.62 \times 10^{57}$, the ratio of the radii of these objects gives the coefficient of similarity in sizes $P=1.4 \times 10^{19}$, and the coefficient of similarity in speeds of same-type processes equals $S=0.23$ . It seems that the ratio $\frac{r_{s}}{r_{p}}$ should equal $P$, but this is not so, because the radius of curvature is derived through the energy density and is not simply a linear dimension. In order to show this, we will take into account that in (27) the scalar curvature inside each object is proportional to the corresponding gravitational constant and mass density:

$$
\frac{r_{s}}{r_{p}}=\sqrt{\frac{G_{a} \rho_{p}}{G \rho_{s}}}=\frac{P}{S}
$$

Meanwhile, according to the dimension theory, the ratio of the gravitational constants is $\frac{G}{G_{a}}=\frac{R S^{2}}{\Phi}$ and the ratio of the mass densities is $\frac{\rho_{s}}{\rho_{p}}=\frac{\Phi}{P^{3}}$.

From the results of Section 4 it follows that inside the proton the cosmological constant, taking into account the strong gravitational constant, should be of the order of $\Lambda_{p} \approx \frac{16 \pi G_{a} \rho_{p}}{c^{2}} \approx 5.1 \times 10^{31} \mathrm{~m}^{-2}$. This is 83 orders of magnitude greater than the cosmological 
constant $\Lambda \approx 10^{-52} \mathrm{~m}^{-2}$, which follows from the general theory of relativity as applied to the observable Universe. In this connection we recall that in cosmology there is still unexplained problem of the cosmological constant. The essence of this problem is that the cosmological constant, calculated with the help of the general theory of relativity for the cosmic space, is almost 120 orders of magnitude less than the cosmological constant for the zero vacuum energy, according to quantum physics. The cosmological constant is a required element in the LambdaCDM model, and in this case it becomes unclear why the expected large magnitude of the zero vacuum energy is transformed into such a small cosmological constant of the cosmic space of the Universe [14].

Our explanation of the problem of the cosmological constant is as follows. Discrepancy between the conclusions of the quantum physics and the general theory of relativity in respect of the cosmological constant is associated with the geometrical approach of the general theory of relativity, which replaces the gravitation, as a force action, with the spacetime curvature. This leads to the absence in this theory of the stress-energy tensor of the gravitational field and to the impossibility of calculating the energy of the internal parts of the physical system under study, which also significantly complicates quantization of the general theory of relativity.

In the covariant theory of gravitation there are no such problems. We find separate components of the energy inside the body, and with their help we determine the corresponding cosmological constant for each body. The observed part of the Universe can be considered as the internal part of some global body, and in this case the cosmological constant is of the order of $\Lambda \approx 10^{-52}$ $\mathrm{m}^{-2}$, besides it is a constant value, which characterizes the entire space filled with stars and galaxies. For the neutron star, the cosmological constant reaches the value of $\bar{\Lambda}_{s} \approx 1.4 \times 10^{-8}$ $\mathrm{m}^{-2}$, and for the proton it equals $\Lambda_{0} \approx 2.2 \times 10^{-8} \mathrm{~m}^{-2}$ for the ordinary gravitation and $\Lambda_{p} \approx 5.1 \times 10^{31} \mathrm{~m}^{-2}$ in the strong gravitational field.

According to the approach to energy and metric gauging in the covariant theory of gravitation, outside the body in the space without matter the cosmological constant and scalar curvature of spacetime vanish. Thus, the alleged relation between the cosmological constant and the zero vacuum energy of the quantum physics outside the body is broken. As for the difference between the cosmological constants inside the proton and the neutron star on the one hand, and the cosmic space on the other hand, it is explained by the fact that the cosmological constant of the observable Universe is the cosmological constant of all the particles and bodies of the Universe averaged over the space. 
We should note that in the modernized Le Sage's model gravitation can arise as a consequence of the action of the fluxes of relativistic particles of the vacuum field on the bodies $[15,16]$. In this case, the standard problems of the Le Sage's model are eliminated by explaining the way in which the vacuum particles interact with the matter [17]. In this case, instead of searching for the quantum zero energy of the vacuum it is possible to determine the energy density of the vacuum field particles, to derive the gravitational constant and the electric constant through the vacuum field parameters and to explain the effect of the Newton's and Coulomb's laws for gravitational and electric forces.

The equations of the covariant theory of gravitation describe only the consequences of interaction of the vacuum field's particles with the matter, which are expressed in changing of the acting forces, changing of the matter energy, as well as in creating inertia of the bodies and imparting mass to them. This means that the energy density of the vacuum field's particles, despite its largeness, is not taken into account as an essential component of the cosmological constant. However, the energy of the vacuum field's particles influences indirectly the magnitude of the cosmological constant inside a particular system through the averaged density of the rest energy of the system's particles.

\section{References}

[1] S. G. Fedosin, About the cosmological constant, acceleration field, pressure field and energy. Jordan Journal of Physics 9(1) (2016) 1-30.

[2] S. G. Fedosin, The metric outside a fixed charged body in the covariant theory of gravitation. International Frontier Science Letters 1(1) (2014) 41-46.

[3] S. G. Fedosin, The integral energy-momentum 4-vector and analysis of 4/3 problem based on the pressure field and acceleration field. American Journal of Modern Physics 3(4) (2014) 152-167.

[4] M. Tegmark, et al (the SDSS collaboration), Cosmological parameters from SDSS and WMAP. Physical Review D 69(10) (2004) 103501-103501.

[5] S. G. Fedosin, The radius of the proton in the self-consistent model. Hadronic Journal 35(4) (2012) 349-363.

[6] A. Salam, J. Strathdee, Confinement through tensor gauge fields. Physical Review D 18 (12) (1978) 4596-4609.

[7] R. L. Oldershaw, Discrete scale relativity. Astrophysics and Space Science 311(4) (2007) 431-433.

[8] S. G. Fedosin, Fizika i filosofiia podobiia ot preonov do metagalaktik. Perm, pages 544 (1999). ISBN 5-8131-0012-1.

[9] V. A. Fock, The theory of space, time and gravitation. Macmillan. (1964).

[10] S. G. Fedosin, Relativistic energy and mass in the weak field limit. Jordan Journal of Physics 8(1) (2015) 1-16.

[11] S. G. Fedosin, The virial theorem and the kinetic energy of particles of a macroscopic system in the general field concept. Continuum Mechanics and Thermodynamics 29(2) (2016) 361-371. 
[12] S. G. Fedosin, Estimation of the physical parameters of planets and stars in the gravitational equilibrium model. Canadian Journal of Physics 94(4) (2016) 370-379.

[13] S. G. Fedosin, The physical theories and infinite hierarchical nesting of matter, Volume 1, LAP LAMBERT Academic Publishing, pages 580 (2014). ISBN 978-3-659-57301-9.

[14] J. Martin, Everything you always wanted to know about the cosmological constant problem (but were afraid to ask). Comptes Rendus Physique 13(6) (2012) 566-665.

[15] S. G. Fedosin, The graviton field as the source of mass and gravitational force in the modernized Le Sage's model. Physical Science International Journal 8(4) (2015) 1-18.

[16] S. G. Fedosin, The force vacuum field as an alternative to the ether and quantum vacuum. WSEAS transactions on applied and theoretical mechanics 10 (2015) 31-38.

[17] S. G. Fedosin, The charged component of the vacuum field as the source of electric force in the modernized Le Sage's model. Journal of Fundamental and Applied Sciences 8(3) (2016) 971-1020. 